\title{
Rapid bacteria selection using Aquaphotomics and near infrared spectroscopy
}

\author{
Zoltan Kovacs, ${ }^{a *}$ Aleksandar Slavchev, ${ }^{\mathrm{b}}$ Gyorgy Bazar, ${ }^{\mathrm{c}}$ Bernhard Pollner ${ }^{\mathrm{d}}$ and Roumiana Tsenkova ${ }^{\mathrm{e}}$ \\ aDepartment of Physics and Control, Faculty of Food Science, Szent Istvan University, Budapest, Hungary. E-mail: Kovacs.Zoltan3@etk.szie.hu \\ bDepartment of Microbiology, University of Food Technologies, Plovdiv, Bulgaria \\ cFaculty of Agricultural and Environmental Sciences, Kaposvar University, Kaposvar, Hungary \\ ${ }^{\mathrm{d} D i v i s i o n}$ of Hygiene and Medical Microbiology, Medical University of Innsbruck, Innsbruck, Austria \\ eBiomeasurement Technology Laboratory, Graduate School of Agricultural Science, Kobe University, Kobe, Japan
}

Efficient, quick and inexpensive screening methods, which provide rapid, in vivo comprehensive probiotic bacteria evaluation, are highly desired in contemporary microbiology. Aquaphotomics is a novel scientific approach for the exploration of aqueous systems through rapid and comprehensive analysis of water-light interaction as a potential source of information for better understanding of the biological world using spectroscopy. The water spectral pattern of the solution as a molecular fingerprint of the cell culture system can be used for quick determination of biological systems. Our objectives are to use near infrared (NIR) spectroscopy with the Aquaphotomics evaluation technique for in vivo discrimination of resistant and non-resistant Lactobacillus strains, and to predict their low $\mathrm{pH}$ and bile resistances using different growth stages and different wavelength ranges. Therefore, NIR spectroscopy with Aquaphotomics was applied to monitor the growth of Lactobacillus bulgaricus, Lactobacillus pentosus and Lactobacillus gasseri bacteria strains. Their growth rate, maximal optical density, low $\mathrm{pH}$ and bile tolerances were measured and used as a reference data for the analysis of the simultaneously acquired spectral data. The acquired NIR spectra were subjected to various multivariate data analyses to build different qualitative and quantitative models to classify the bacteria strains with different probiotic strength and to determine their phenotypic characteristics. The results of the in vivo evaluation of probiotic and nonprobiotic bacteria strains provided accurate, fast and non-invasive identification of probiotic bacteria strains based on spectral monitoring of their bacterial growth. Results also proved that the prediction of the main phenotypic characteristics of probiotic candidates is also possible with NIR spectroscopy and Aquaphotomics.

\section{Introduction}

Recently, the market of functional food has been growing significantly as people started to pay more attention to healthy food consumption. Products containing probiotic are an important part of the functional food diets. Probiotic bacteria are non-pathogenic microorganisms that, when ingested in sufficient viable numbers, confer a health benefit on the host. ${ }^{1}$ Positive effects of probiotics include alleviation of irritable bowel syndrome and inflammatory bowel diseases, ${ }^{2}$ harmonising of the gastro-intestinal tract microflora and strengthens the immune system ${ }^{3}$ or decrease the symptoms of allergies in receptive individuals. ${ }^{4}$ These beneficial bacteria must reach the colon in relatively high viable cell counts to employ their positive effect. Therefore, they must survive harsh conditions, such as low $\mathrm{pH}$, presence of pepsin and high concentration of bile salt. ${ }^{5}$ The best probiotic candidates are the strains which can gain a high amount of biomass in a short period of time and have good ability to survive the harsh environment. The selection of bacteria possessing strong probiotic charac teristics is a major problem. The currently applied methods based on particular genes selection and in vitro examination of strain growth under model conditions ${ }^{5}$ are time-

\section{Correspondence}

Z. Kovacs (Kovacs.Zoltan3@etk.szie.hu)

doi: $10.1255 /$ nir2017.065

Citation: Z. Kovacs, A. Slavchev, G. Bazar, B. Pollner and R. Tsenkova, "Rapid bacteria selection using Aquaphotomics and near infrared spectroscopy", in Proc. $18^{\text {th }}$ Int. Conf. Near Infrared Spectrosc., Ed by S.B. Engelsen, K.M. Sørensen and F. van den Berg. IM Publications Open, Chichester, pp. $65-69$ (2019). https://doi.org/10.1255/nir2017.065

\section{(c) 2019 The Authors}

This licence permits you to use, share, copy and redistribute the paper in any medium or any format provided that a full citation to the original paper is given, the use is not for commercial purposes and the paper is not changed in any way.

ISBN: 978-1-906715-27-4 
consuming, expensive and not always give satisfactory results. Therefore, development of efficient screening method coupled with cell functionality evaluation is highly needed in contemporary microbiology. ${ }^{6}$ In recent years, a new approach called "aquaphotomics" has been proposed for the examination of biological systems, using their water spectra as a molecular mirror. ${ }^{7,8}$ Aquaphotomics has been successfully applied for identification and discrimination of bacterial species at very low concentrations. ${ }^{9}$ Water spectral patterns of the living microorganisms provide information about their functionality and can be used as fingerprint of cells phenotype. Therefore, Aquaphotomics has the potential to replace the phenotypic and genetic approach for probiotic bacteria selection.

Thus, the objective of this research was to evaluate the applicability of near infrared (NIR) spectroscopy and Aquaphotomics for in vivo discrimination of resistant and non-resistant Lactobacillus strains, i.e. strains possessing different probiotic properties (probiotic, moderate and non-probiotic). An additional aim was to build quantitative models for the prediction of the main phenotypic characteristics using different wavelength ranges.

\section{Materials and methods}

Seven probiotic and twelve non-probiotic (including six moderate) strains (genus Lactobacillus) were used for the experiments: probiotic strains are L. bulgaricus S06, S10, S11, S19, S22, S25 and L. gasseri S20, moderate strains are L. bulgaricus S01, S07, S08, S09, Y12 and S28, and non-probiotic strains are L. bulgaricus SO2, S03, SO4, S05, S29 and S30.

The strains were provided by "Selur Pharma" Ltd (Bulgaria), except for the strain L. bulgaricus Y12 which was isolated from yoghurt. All microorganisms were freeze-dried and kept at $-80^{\circ} \mathrm{C}$ before the experiments. The three groups (non-probiotic, moderate and probiotic) were determined based on the main phenotypic characteristics of the strains by means of their growth rate, biomass production, minimal inhibitory concentration of bile, and best recovery after $3 \mathrm{~h}$ at low $\mathrm{pH}$ and pepsin. The methods used for the determination of the main phenotypic characteristics of the strains is described in detail elsewhere. ${ }^{6}$

Lactobacillus strains were cultivated in MRS at $37^{\circ} \mathrm{C}$ for $24 \mathrm{~h}$ with shaking in $50 \mathrm{~mL}$ centrifuge tubes. NIR trans- flectance spectra $(400-2500 \mathrm{~nm}, 0.5 \mathrm{~nm}$ steps, $0.5 \mathrm{~mm}$ layer thickness) of the cultures were collected every 4 min using a XDS OptiProbe Analyzer, immersion probe (FOSS NIRSystems). A reference spectrum was taken at the beginning of every measurement series placing the immersion probe in the dark aperture position of the instrument. The spectra taken in the first $40 \mathrm{~min}$ of the cultivation time were discarded and those after 40 min until the scan of $20 \mathrm{~h}$ were used for data evaluation. The total number of spectra in the evaluation was 5700 (19 strains $\times 300$ spectra). Spectra acquisition was performed with VISION 3.50 (FOSS NIRSystems, Inc., Hoganas, Sweden) software.

The spectra were pretreated using Savitzky-Golay smoothing ( $2^{\text {nd }}$ order polynomial and 21 points) and multiplicative scatter correction (MSC) before the multidimensional data evaluation. Different spectral ranges were used separately during the evaluation $(640-840 \mathrm{~nm}$, $840-1100 \mathrm{~nm}, 1300-1600 \mathrm{~nm}, 1100-1850 \mathrm{~nm})$ to find the best interval for the discrimination of the different strains. Principal Component Analysis (PCA) was used to describe multidimensional patterns of the NIR dataset and to discover outliers and to provide orthogonal variables for Linear Discriminant Analysis (LDA) where the first seven PCs were used as input to LDA. Moving Window LDA (MWLDA) was used to find the optimal time window for classification of the three main groups where windows of 20 spectra at a time were included in a single LDA model, moving one spectrum forward to build the next model. The performance of LDA models were evaluated by the correct classification rate during training, onestrain-out cross-validation and independent prediction. Partial Least Square Regression (PLSR) models were built based on the time window showing the best classification of the main groups to predict the main phenotypic characteristics of the strains. Data of 15 strains was used for model building and data of four strains (S19, S25, S08 and S05) was applied for testing.

\section{Results and discussion Classification of non-probiotic, moderate and probiotic Lactobacillus strains}

Results of the correct classification rate observed during the independent tests at every time points of the MWLDA models built on the different spectral ranges are summarised in Figure 1. The growing curve of L. bulgaricus SO6 


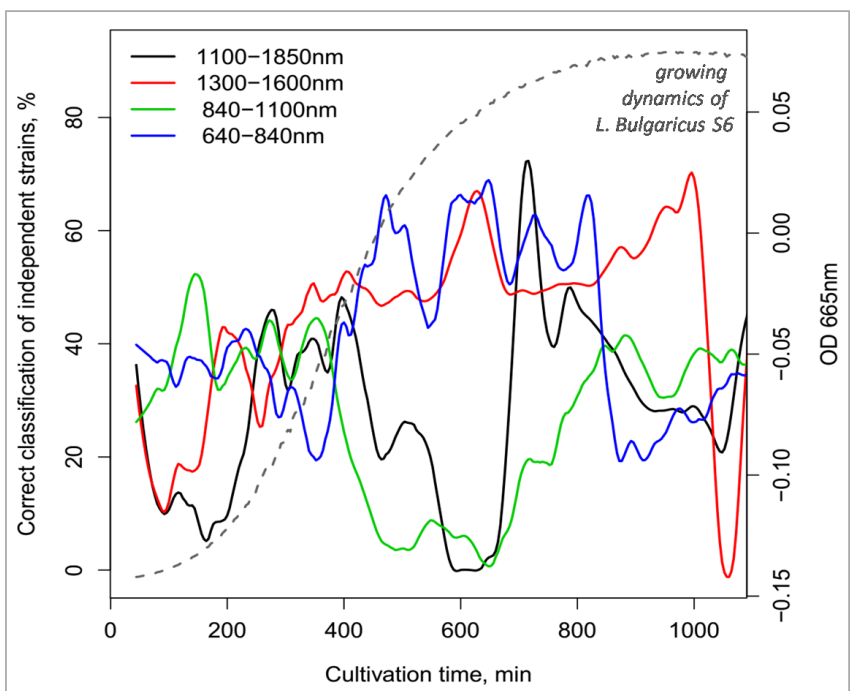

Figure 1. Correct classification achieved with MWLDA using different wavelength ranges showing the best cultivation time for classification after $600 \mathrm{~min}$.

is also shown to depict the growing dynamics of a strain. The best classification among the three main groups was found at the end of the exponential growth phase using the wavelength ranges $1100-1850 \mathrm{~nm}, 1300-1600 \mathrm{~nm}$ and 640-840 nm.

The correct classification rates during training, onestrain-out cross-validation and independent prediction of the best LDA models found for the different spectral ranges are shown in Figure 2 together with the respective time windows. Although the wavelength range between
$640 \mathrm{~nm}$ and $840 \mathrm{~nm}$ provided slightly different correct classification compared to the ranges of $1100-1850 \mathrm{~nm}$ and $1300-1600 \mathrm{~nm}$ during the independent test, the later ones resulted in $100 \%$ correct classification in training and cross-validation.

LDA score plots of the classification models built for the classification of probiotic, moderate and non-probiotic bacteria strains in the optimal time window are shown in Figure 3 for $1100-1850 \mathrm{~nm}$ and $1300-1600 \mathrm{~nm}$ spectral ranges. As the $100 \%$ correct classification rate in training and cross-validation indicated the confidence ellipses of the different strain phenotypes are not overlapping. Scores of the strains used for independent prediction are projected out of the confidence ellipses, but scores of probiotic strains are closer to the group of moderate ones than to the groups of non-probiotic types and vice versa, i.e. misclassification was observed only between groups of probiotic and moderate strains and between groups of non-probiotic and moderate ones.

\section{Prediction of the phenotypic characteristics of the Lactobacillus strains}

Results of the PLSR models built to determine a relationship between spectral data and main phenotypic characteristics of Lactobacillus strains using the best time window for $1100-1850 \mathrm{~nm}$ and $1300-1600 \mathrm{~nm}$ spectral intervals are summarised in Table 1.

Determination coefficients of cross-validation show closer correlation between the measured and predicted

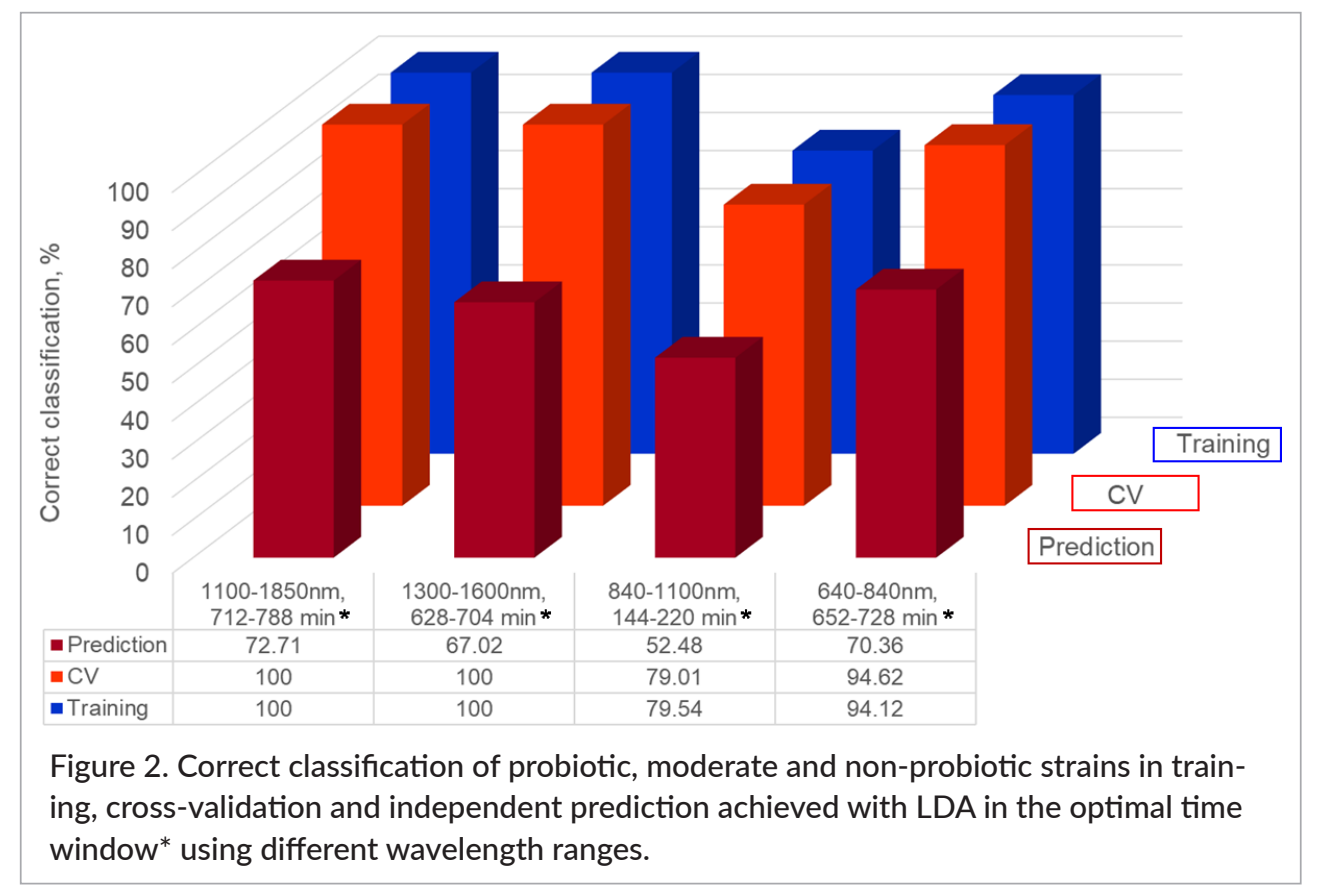



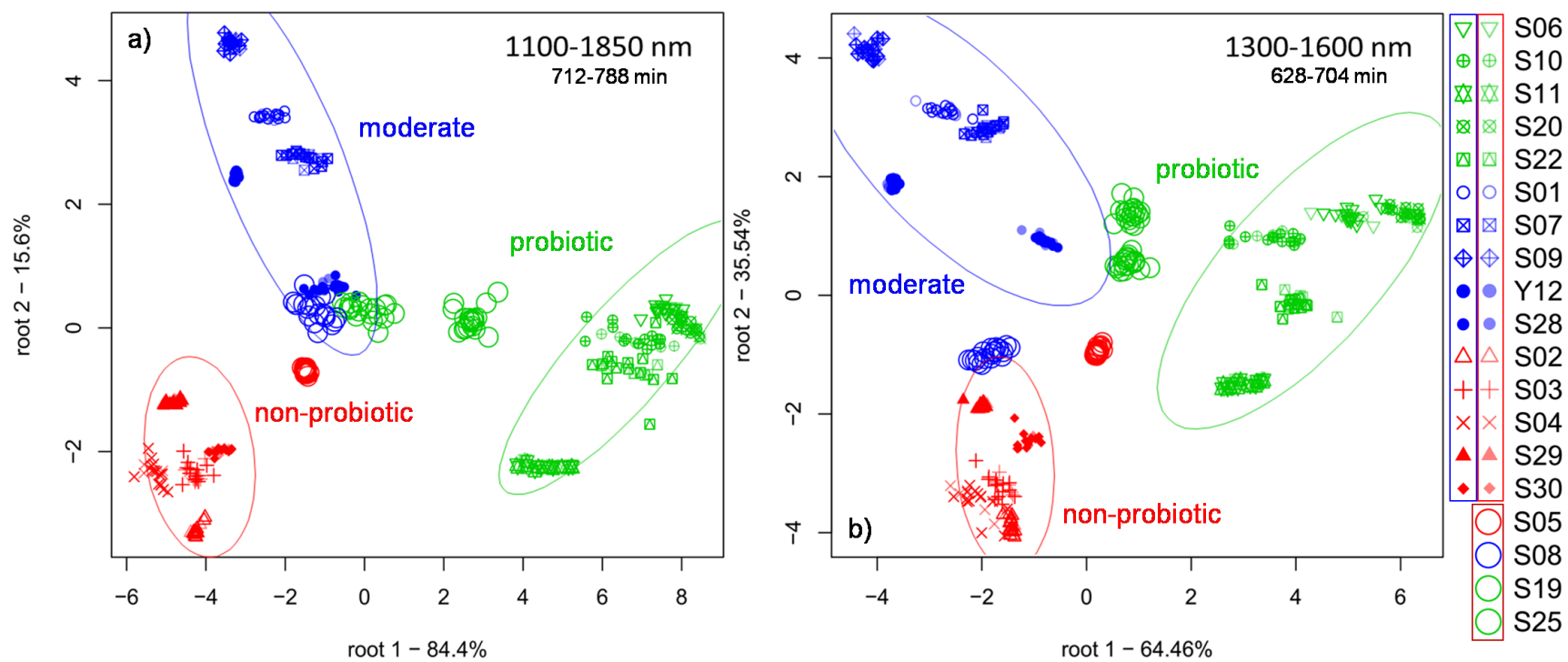

Figure 3. LDA score plots with $95 \%$ confidence ellipses presenting the classification of probiotic, moderate and non-probiotic strains in the optimal time window, a) $1100-1850 \mathrm{~nm}$, b) $1300-1600 \mathrm{~nm}$.

phenotypic characteristics for maximal optical density, low pH tolerance and bile tolerance for the wavelength range between $1100 \mathrm{~nm}$ and $1850 \mathrm{~nm}$ than for 1300$1600 \mathrm{~nm}$. However, average prediction error of the independent test has been found lower for all the tested phenotypic characteristics using the spectral range between $1300 \mathrm{~nm}$ and $1600 \mathrm{~nm}$.

\section{Conclusions}

The evaluation of the different wavelength ranges and different time windows of the cultivation time for classification of groups of probiotic, moderate and non-probiotic Lactobacillus bacteria strains have been performed. Results showed spectral ranges between $1100 \mathrm{~nm}$ and $1850 \mathrm{~nm}$ and between $1300 \mathrm{~nm}$ and $1600 \mathrm{~nm}$ in the time

Table 1. Summary of PLSR models' accuracy built to predict main phenotypic characteristics of Lactobacillus strains. OD: optical density.

\begin{tabular}{|c|c|c|c|c|}
\hline $\begin{array}{l}n_{\text {training }}=300 \\
n_{\text {prediction }}=80\end{array}$ & Growth rate & $\begin{array}{l}\text { Maximal optical } \\
\text { density }\end{array}$ & $\begin{array}{l}\text { Low } \mathrm{pH} \\
\text { tolerance }\end{array}$ & Bile tolerance \\
\hline \multicolumn{5}{|c|}{ Wavelength range: $1100-1850 \mathrm{~nm}$, Optimal time window: 712-788 min } \\
\hline Factor\# & 8 & 6 & 5 & 7 \\
\hline $\mathrm{R}_{\mathrm{Cal}}^{2}$ & 0.9564 & 0.9201 & 0.9452 & 0.9716 \\
\hline RMSEC & 0.0160 & 0.2076 & 0.0103 & 0.1559 \\
\hline$R_{C V}^{2}$ & 0.9560 & 0.9198 & 0.9449 & 0.9711 \\
\hline RMSECV & 0.0161 & 0.2079 & 0.0103 & 0.1571 \\
\hline RMSEP & 0.0768 & 0.4083 & 0.0385 & 0.4922 \\
\hline \multicolumn{5}{|c|}{ Wavelength range: $1300-1600 \mathrm{~nm}$, Optimal time window: 628-704 min } \\
\hline Factor\# & 9 & 5 & 5 & 7 \\
\hline $\mathrm{R}_{\mathrm{Cal}}^{2}$ & 0.9755 & 0.8125 & 0.8514 & 0.8816 \\
\hline RMSEC & 0.0120 & 0.3180 & 0.0169 & 0.3182 \\
\hline $\mathrm{R}_{\mathrm{CV}}^{2}$ & 0.9720 & 0.8123 & 0.8512 & 0.8811 \\
\hline RMSECV & 0.0128 & 0.3182 & 0.0169 & 0.3190 \\
\hline RMSEP & 0.0743 & 0.3917 & 0.0338 & 0.4307 \\
\hline
\end{tabular}


windows between $712 \mathrm{~min}$ and $788 \mathrm{~min}$ and between $628 \mathrm{~min}$ and $704 \mathrm{~min}$, respectively, are the best for the classification of the three main groups. The results of the in vivo evaluation of bacteria strains provided accurate, fast and non-invasive identification of the different strains based on spectral monitoring of their bacterial growth. Results also proved the prediction of the main phenotypic characteristics of probiotic candidates is also possible with NIR spectroscopy and Aquaphotomics. The multivariate methods applied for the spectral data assessment with regards to phenotype identification showed several common water absorbance bands with high importance.

\section{Acknowledgements}

This study was supported by the János Bolyai Research Scholarship of the Hungarian Academy of Sciences, Hungary (Zoltan Kovacs) and by the New National Excellence Program of the Ministry of Human Capacities (ÚNKP-16-4, Gyorgy Bazar). The authors would like to thank Selur Pharma Ltd, Bulgaria for providing the bacterial strains.

\section{References}

1. FAO/WHO, Joint Working Group Report on Drafting Guidelines for the Evaluation of Probiotic in Food. London, Ontario, Canada, April 30 and May 1, London (2002).

2. L.V. McFarland, "Meta-analysis of probiotics for the prevention of traveler's diarrhea", Travel Med. Infect. Dis. 5(2), 97-105 (2007). https://doi.org/10.1016/j. tmaid.2005.10.003

3. S. Parvez, K.A. Malik, S. Ah Kang and H.-Y. Kim, "Probiotics and their fermented food products are beneficial for health", J. Appl. Microbiol. 100(6), 1171-1185 (2006). https://doi.org/10.1111/j.13652672.2006.02963.x

4. M.A. Kalliomäki and E. Isolauri, "Probiotics and down-regulation of the allergic response", Immunol. Allergy Clinics N. Amer. 24(4), 739-752, viii (2004). https://doi.org/10.1016/j.iac.2004.06.006

5. I. Pitino, C.L. Randazzo, G. Mandalari, A. Lo Curto, R.M. Faulks, Y. Le Marc, C. Bisignano, C. Caggia and M.S.J. Wickham, "Survival of Lactobacillus rhamnosus strains in the upper gastrointestinal tract", Food Microbiol. 27(8), 1121-1127 (2010). https://doi. org/10.1016/j.fm.2010.07.019

6. A. Slavchev, Z. Kovacs, H. Koshiba, A. Nagai, G. Bázár, A. Krastanov, Y. Kubota and R. Tsenkova, "Monitoring of water spectral pattern reveals differences in probiotics growth when used for rapid bacteria selection", PLoS One 10(7), e0130698 (2015). https://doi.org/10.1371/journal.pone.0130698

7. B. Jinendra, K. Tamaki, S. Kuroki, M. Vassileva, S. Yoshida and R. Tsenkova, "Near infrared spectroscopy and aquaphotomics: Novel approach for rapid in vivo diagnosis of virus infected soybean", Biochem. Biophys. Res. Commun. 397(4), 685-690 (2010). https://doi.org/10.1016/j.bbrc.2010.06.007

8. R. Tsenkova, "Aquaphotomics: dynamic spectroscopy of aqueous and biological systems describes peculiarities of water", J. Near Infrared Spectrosc. 17(1), 303 (2009). https://doi.org/10.1255/jnirs.869

9. Y. Nakakimura, M. Vassileva, T. Stoyanchev, K. Nakai, R. Osawa, J. Kawano and R. Tsenkova, "Extracellular metabolites play a dominant role in near-infrared spectroscopic quantification of bacteria at foodsafety level concentrations", Anal. Methods 4(5), 1389 (2012). https://doi.org/10.1039/c2ay05771a 